Typeset with jpsj2.cls $<$ ver.1.1.1 $>$

\title{
A Recursive Method of the Stochastic State Selection for Quantum Spin Systems
}

\author{
Tomo Munehisa and Yasuko Munehisa \\ Faculty of Engineering, Univ. of Yamanashi, Kofu 400-8511
}

(Received December 22, 2018)

In this paper we propose the recursive stochastic state selection method, an extension of the recently developed stochastic state selection method in Monte Carlo calculations for quantum spin systems. In this recursive method we use intermediate states to define probability functions for stochastic state selections. Then we can diminish variances of samplings when we calculate expectation values of the powers of the Hamiltonian. In order to show the improvement we perform numerical calculations of the spin- $1 / 2$ anti-ferromagnetic Heisenberg model on the triangular lattice. Examining results on the ground state of the 21-site system we confide this method in its effectiveness. We also calculate the lowest and the excited energy eigenvalues as well as the static structure factor for the 36-site system. The maximum number of basis states kept in a computer memory for this system is about $3.6 \times 10^{7}$. Employing a translationally invariant initial trial state, we evaluate the lowest energy eigenvalue within $0.5 \%$ of the statistical errors.

KEYWORDS: quantum spin, large size, numerical calculation, Monte Carlo, triangular lattice

\section{Introduction}

Quite recently we have proposed a new method of Monte Carlo calculations, which we call the stochastic state selection (SSS) method, ${ }^{1)}$ to obtain energy eigenvalues of quantum spin systems. Under this method we can numerically eliminate a lot of basis states in a mathematically justified way. This reduction enables us to calculate expectation values of powers of the Hamiltonian even when a limited computer memory is available. In a previous work ${ }^{2)}$ we applied this method to the two-dimensional Shastry-Sutherland model and obtained reliable energy eigenvalues for the first excited states on a $8 \times 8$ lattice across the critical region.

In this paper we propose an extended version of the SSS method, which we will refer to as the recursive stochastic state selection (RSSS) method. In the SSS method we have introduced random choice matrices, whose elements are stochastic variables to follow probability functions named on-off probability functions. Here these probability functions are defined only once at the beginning. In contrast to them, the on-off probability functions in the RSSS method are defined in sequence for each random choice matrix. By this extension we can expect smaller variances of the expectation values of high powers of the Hamiltonian compared to the SSS results. Here we examine the effectiveness of the RSSS method in numerical studies of the anti-ferromagnetic Heisenberg quantum spin- $1 / 2$ system on the triangular lattice. ${ }^{3-9)}$ To this typical strongly frustrated system, 
other methods such as the ordinary Monte Carlo methods or the perturbative calculations are hardly applicable. We first show that the method is successful for the model on the lattice of 21 sites. Then we proceed to the 36 -site system to demonstrate that we can estimate the ground state energy eigenvalue, a first-excited energy eigenvalue and the static structure factor by this method.

The plan of this paper is as follows. In the next section we briefly summarize the SSS method in order to prepare for the following section and the Appendix. Section 3 is to explain the RSSS method, where the recursive procedure of the method is stated. In sections 4 and 5 we apply the method to the anti-ferromagnetic Heisenberg spin- $1 / 2$ system on the triangular lattice. Section 4 is devoted to basic examinations. We first examine results on the energy eigenvalue of the ground state for a small system of 21 sites. We show that the RSSS results are in good agreement with exact values. We also observe much less variances for the expectation values of high powers of the Hamiltonian compared to the SSS results. Then we calculate the lowest energy eigenvalue for the 36-site system. Here we discuss on a way to find a good approximate state. In addition, we state how we estimate the ground state energy eigenvalue from numerically calculated expectation values. In section 5 we present further calculations for the 36 -site system. Here we employ an improved initial trial state which reflects the translational invariance of the Hamiltonian. We show that the statistical errors of the ground state energy are much reduced by this improvement.

Our result is $E=-20.04 \pm \begin{aligned} & 0.10 \\ & 0.06\end{aligned}$, which is about $0.6 \%$ higher than the known exact value $E=-20.17344 .^{5,7,8)}$ We also present results on the energy eigenvalue of an excited state and the static structure factor. The final section is devoted to summary and discussions. In the Appendix we discuss some properties concerning to the on-off probability functions.

\section{Stochastic State Selection Method}

This section is devoted to a brief summary of the SSS method. ${ }^{1)}$ Let $|\psi\rangle$ be a state of the system which is expanded by a basis $\{|i\rangle\}$ as

$$
|\psi\rangle=\sum_{i=1}^{N_{\mathrm{V}}}|i\rangle c_{i}
$$

$N_{\mathrm{V}}$ being the size of the full vector space. We denote the Hamiltonian of the system by $\hat{H}$. In order to numerically evaluate the expectation values

$$
E(L) \equiv\left\langle\psi\left|\hat{H}^{L}\right| \psi\right\rangle \quad(L=1,2, \cdots)
$$

we define ${ }^{10)}$

$$
E_{\{\eta\}}(L) \equiv\left\langle\psi\left|\hat{H} M_{\left\{\eta^{(L)}\right\}} \hat{H} M_{\left\{\eta^{(L-1)}\right\}} \cdots \hat{H} M_{\left\{\eta^{(1)}\right\}}\right| \psi\right\rangle
$$


The random choice matrices $M_{\left\{\eta^{(m)}\right\}}(m=1,2, \cdots, L)$ are the following diagonal matrices

$$
M_{\left\{\eta^{(m)}\right\}} \equiv\left(\begin{array}{cccc}
\eta_{1}^{(m)} & 0 & \cdots & 0 \\
0 & \eta_{2}^{(m)} & \ldots & 0 \\
\cdots & \cdots & \cdots & \cdots \\
0 & 0 & \cdots & \eta_{N_{\mathrm{V}}}^{(m)}
\end{array}\right),
$$

with random variables $\eta_{i}^{(m)}$ s. These variables are determined according to the on-off probability functions

$$
P_{i}(\eta) \equiv P\left(\eta ; a_{i}\right), \quad a_{i} \equiv \begin{cases}\max \left(1, \epsilon /\left|c_{i}\right|\right) & \left(c_{i} \neq 0\right) \\ \epsilon / \delta & \left(c_{i}=0\right)\end{cases}
$$

where

$$
P(\eta ; a) \equiv \frac{1}{a} \delta(\eta-a)+\left(1-\frac{1}{a}\right) \delta(\eta)
$$

and $\epsilon$ and $\delta(<\epsilon)$ denote given positive constants. Note that each random choice matrix is independently generated using the same on-off probability function $P_{i}(\eta)$ for $\eta_{i}^{(m)}$. We can reduce the effective number of the basis by operating $M_{\left\{\eta^{(m)}\right\}}$ to the state $\hat{H} M_{\left\{\eta^{(m-1)}\right\}} \cdots \hat{H} M_{\left\{\eta^{(1)}\right\}}|\psi\rangle$ because many of generated values of $\eta_{i}^{(m)}$ 's in $\left\{\eta^{(m)}\right\} \equiv\left\{\eta_{1}^{(m)}, \eta_{2}^{(m)}, \cdots, \eta_{N_{\mathrm{V}}}^{(m)}\right\}$ are zero. It is guaranteed that the statistical average of $E_{\{\eta\}}(L)$ gives $E(L)$,

$$
\left\langle\left\langle E_{\{\eta\}}(L)\right\rangle\right\rangle=E(L),
$$

since the statistical average of $\eta_{i}^{(m)}$ is

$$
\left\langle\left\langle\eta_{i}^{(m)}\right\rangle \equiv \int_{0}^{\infty} \eta_{i}^{(m)} P_{i}\left(\eta_{i}^{(m)}\right) d \eta_{i}^{(m)}=1\right.
$$

and $\left\langle\left\langle\eta_{i}^{(1)} \eta_{j}^{(2)} \cdots \eta_{l}^{(L)}\right\rangle\right\rangle=\left\langle\left\langle\eta_{i}^{(1)}\right\rangle\right\rangle\left\langle\left\langle\eta_{j}^{(2)}\right\rangle\right\rangle \cdots\left\langle\left\langle\eta_{l}^{(L)}\right\rangle\right\rangle$ holds for independently generated $\left\{\eta^{(1)}\right\},\left\{\eta^{(2)}\right\}$, $\cdots,\left\{\eta^{(L)}\right\}$.

\section{Recursive Stochastic State Selection Method}

In this section we show a procedure to estimate expectation values following the RSSS method. While the on-off probability functions (5) in the SSS method are common to all $m$ for each $i$, we generate the random variables $\left\{\eta^{(m)}\right\}$ in the RSSS method using the preceding intermediate state $\hat{H} M_{\left\{\eta^{(m-1)}\right\}} \cdots \hat{H} M_{\left\{\eta^{(1)}\right\}}|\psi\rangle$.

We start from the state $\left|\psi^{(0)}\right\rangle \equiv|\psi\rangle$,

$$
\left|\psi^{(0)}\right\rangle=\sum_{i=1}^{N_{\mathrm{V}}}|i\rangle c_{i}^{(0)}
$$


Then we determine the first random choice matrix $M_{\left\{\eta^{(1)}\right\}}$ generating random variables $\eta_{i}^{(1)}$ according to the on-off probability functions

$$
P_{i}^{(1)}(\eta) \equiv P\left(\eta ; a_{i}^{(1)}\right), \quad a_{i}^{(1)} \equiv \begin{cases}\max \left(1, \epsilon /\left|c_{i}^{(0)}\right|\right) & \left(c_{i}^{(0)} \neq 0\right) \\ \infty & \left(c_{i}^{(0)}=0\right)\end{cases}
$$

where the function $P(\eta ; a)$ is given by $(6)$ and $\epsilon$ is a positive constant. Calculating the state $\hat{H} M_{\left\{\eta^{(1)}\right\}}\left|\psi^{(0)}\right\rangle$ with this $M_{\left\{\eta^{(1)}\right\}}$ we obtain $\left|\psi^{(1)}\right\rangle \equiv \hat{H} M_{\left\{\eta^{(1)}\right\}}\left|\psi^{(0)}\right\rangle / C^{(1)}$. Here $C^{(1)}(>0)$ is given by the equation

$$
\left[C^{(1)}\right]^{2}=\left\langle\psi^{(0)}\left|M_{\left\{\eta^{(1)}\right\}} \hat{H}^{2} M_{\left\{\eta^{(1)}\right\}}\right| \psi^{(0)}\right\rangle
$$

which follows from the normalization condition $\left\langle\psi^{(1)} \mid \psi^{(1)}\right\rangle=1$. Next we determine the second random choice matrix $M_{\left\{\eta^{(2)}\right\}}$ in the same manner using $\left|\psi^{(1)}\right\rangle=\sum|i\rangle c_{i}^{(1)}$ instead of $\left|\psi^{(0)}\right\rangle$, namely using the probability functions

$$
P_{i}^{(2)}(\eta) \equiv P\left(\eta ; a_{i}^{(2)}\right), \quad a_{i}^{(2)} \equiv\left\{\begin{array}{ll}
\max \left(1, \epsilon /\left|c_{i}^{(1)}\right|\right) & \left(c_{i}^{(1)} \neq 0\right) \\
\infty & \left(c_{i}^{(1)}=0\right)
\end{array} .\right.
$$

Then we proceed to calculate $\hat{H} M_{\left\{\eta^{(2)}\right\}}\left|\psi^{(1)}\right\rangle, C^{(2)}$ and $\left|\psi^{(2)}\right\rangle$. Repeating this procedure we obtain the $m$-th normalized state $\left|\psi^{(m)}\right\rangle$ from the preceding state $\left|\psi^{(m-1)}\right\rangle$,

$$
\begin{aligned}
\left|\psi^{(m)}\right\rangle & \equiv \hat{H} M_{\left\{\eta^{(m)}\right\}}\left|\psi^{(m-1)}\right\rangle / C^{(m)}, \\
{\left[C^{(m)}\right]^{2} } & =\left\langle\psi^{(m-1)}\left|M_{\left\{\eta^{(m)}\right\}} \hat{H}^{2} M_{\left\{\eta^{(m)}\right\}}\right| \psi^{(m-1)}\right\rangle .
\end{aligned}
$$

The on-off probability functions to generate $M_{\left\{\eta^{(m+1)}\right\}}$ are defined using the coefficients $c_{i}^{(m)}$ in $\left|\psi^{(m)}\right\rangle$

$$
\left|\psi^{(m)}\right\rangle=\sum|i\rangle c_{i}^{(m)}
$$

as

$$
P_{i}^{(m+1)}(\eta) \equiv P\left(\eta ; a_{i}^{(m+1)}\right), \quad a_{i}^{(m+1)} \equiv\left\{\begin{array}{ll}
\max \left(1, \epsilon /\left|c_{i}^{(m)}\right|\right) & \left(c_{i}^{(m)} \neq 0\right) \\
\infty & \left(c_{i}^{(m)}=0\right)
\end{array} .\right.
$$

Thus in the RSSS method we obtain for $E(L)_{\{\eta\}}$ defined by (3),

$$
E(L)_{\{\eta\}}=\left\langle\psi^{(0)} \mid \psi^{(L)}\right\rangle C^{(L)} C^{(L-1)} \cdots C^{(1)}
$$

for each set of the random values of $\{\eta\}=\left\{\left\{\eta^{(L)}\right\}, \cdots,\left\{\eta^{(1)}\right\}\right\}$.

Note that we should be careful here to think of the statistical averages concerning $\left\{\eta^{(m)}\right\}$ because the probability functions $P_{i}^{(m)}(\eta)$ 's depend on the precedingly generated sets of random 
variables $\left\{\eta^{(1)}\right\},\left\{\eta^{(2)}\right\}, \cdots,\left\{\eta^{(m-1)}\right\}$. Yet, we see that $\left\langle\left\langle\eta_{i}^{(m)}\right\rangle\right\rangle=1$ holds even in this case for a fixed set of $\left\{\left\{\eta^{(m-1)}\right\}, \cdots,\left\{\eta^{(1)}\right\}\right\}$. We therefore obtain the relation (7) if we do recursive samplings which ensure

$$
\left.\left\langle\left\langle\left\langle\left\langle\cdots\left\langle\left\langle\left\langle\eta_{i}^{(L)}\right\rangle\right\rangle_{\text {fixed }\left\{\left\{\eta^{(L-1)}\right\}, \cdots,\left\{\eta^{(1)}\right\}\right\}} \eta_{j}^{(L-1)}\right\rangle\right\rangle_{\text {fixed }\left\{\left\{\eta^{(L-2)}\right\}, \cdots,\left\{\eta^{(1)}\right\}\right\}} \cdots \eta_{k}^{(2)}\right\rangle\right\rangle_{\text {fixed }\left\{\eta^{(1)}\right\}} \eta_{l}^{(1)}\right\rangle\right\rangle=1
$$

We expect that variances of $\left\langle\left\langle E_{\{\eta\}}(L)\right\rangle\right\rangle$ in the RSSS method become smaller for large $L$ than those in the SSS method because our definition of $a_{i}^{(m+1)}$ in (16) minimizes a quantity $S^{(m)} \equiv$ $\epsilon^{2}\left\langle\left\langle N^{(m)}\right\rangle\right\rangle+\left\langle\left\langle\left[g^{(m)}\right]^{2}\right\rangle\right\rangle$. Here $g^{(m)}$ measures the difference of the truncated state $M_{\left\{\eta^{(m+1)}\right\}}\left|\psi^{(m)}\right\rangle$ from the original one $\left|\psi^{(m)}\right\rangle$,

$$
\left|\chi^{(m)}\right\rangle g^{(m)} \equiv M_{\left\{\eta^{(m+1)}\right\}}\left|\psi^{(m)}\right\rangle-\left|\psi^{(m)}\right\rangle, \quad\left\langle\chi^{(m)} \mid \chi^{(m)}\right\rangle=1
$$

and $N^{(m)}$ denotes the number of non-zero coefficients in (15). The reason why $S^{(m)}$ is minimized by $a_{i}^{(m+1)}$ in (16) will be given in the Appendix.

\section{Numerical Examinations}

Here we numerically examine our method. We measure

$$
\left\langle\left\langle E_{\{\eta\}}(L)\right\rangle\right\rangle_{\mathrm{smpl}} \equiv \frac{1}{n_{\mathrm{smpl}}} \sum_{k=1}^{n_{\mathrm{smpl}}} E_{\{\eta\}_{k}}(L),
$$

in order to evaluate $E(L)$, where $\{\eta\}_{k}$ denotes the $k$-th set of random values of $\left\{\left\{\eta^{(L)}\right\}, \cdots,\left\{\eta^{(1)}\right\}\right\}$. In the SSS method, replacing $\left\langle\left\langle E_{\{\eta\}}(L)\right\rangle\right\rangle$ by $(20)$ is theoretically allowed because $\left\{\eta^{(m)}\right\}$ is independent of $\left\{\eta^{\left(m^{\prime}\right)}\right\}$ if $m \neq m^{\prime}$. In the RSSS method, on the other hand, things are more complicated as was pointed out in the previous section. Since the recursive sampling to ensure (18) is difficult to carry out in numerical work because of the bursting sample numbers for large values of $L$, we adopt a sequential generation of only one set of $\{\eta\}_{k}=\left\{\left\{\eta^{(L)}\right\}, \cdots,\left\{\eta^{(1)}\right\}\right\}_{k}$ for each sampling in (20). This replacement is analytically justified in the sense that an iterated integral is replaceable by a multiple integral in most cases. We will see our numerical check confirms that we can obtain reliable values of $E(L)$ by (20) without recursive samplings.

After the same manner as the SSS method, we evaluate the error of $\left\langle\left\langle E_{\{\eta\}}(L)\right\rangle\right\rangle_{\text {smpl }}$ by

$$
\operatorname{Er}(L) \equiv 2 \sqrt{\frac{\rho_{\{\eta\}}^{2}(L)}{n_{\mathrm{smpl}}}},
$$

where

$$
\rho_{\{\eta\}}^{2}(L) \equiv\left\langle\left\langle\left[E_{\{\eta\}}(L)\right]^{2}\right\rangle\right\rangle_{\mathrm{smpl}}-\left[\left\langle\left\langle E_{\{\eta\}}(L)\right\rangle\right\rangle_{\mathrm{smpl}}\right]^{2}
$$


As a concrete example we adopt the anti-ferromagnetic Heisenberg quantum spin system on the triangular lattice. The Hamiltonian of the model is

$$
\hat{H}=\frac{J}{4} \sum_{(i, j)} \boldsymbol{\sigma}_{i} \cdot \boldsymbol{\sigma}_{j},
$$

where $\boldsymbol{\sigma}_{i}$ is the Pauli matrix on the site $i$ and $(i, j)$ runs over all nearest neighbor pairs on the lattice with the periodic boundary conditions for both directions. ${ }^{7,11)}$ The coupling $J$ is fixed to be 1 throughout this paper.

First we present in Tables I and II our results for a small lattice with 21 sites. Since we can obtain an exact ground state $\left|\psi_{\mathrm{E}}\right\rangle$ by the Lanczos method for this system, we put $\left|\psi^{(0)}\right\rangle=\left|\psi_{\mathrm{E}}\right\rangle$ here with $N^{(0)}=352,716$. Table I shows results on $\left\langle\left\langle E_{\mathrm{E}\{\eta\}}(L)\right\rangle\right\rangle_{\text {smpl }}$ up to $L=15$ together with the exact values $E_{\mathrm{E}}(L)$, where we add a suffix $\mathrm{E}$ in order to indicate that an exact eigenstate is used as $\left|\psi^{(0)}\right\rangle$. The value of the parameter $\epsilon$ is 0.1 . In Table II we present $\left\langle\left\langle N_{\mathrm{b}}^{\mathrm{E}}(L)\right\rangle\right\rangle_{\text {smpl }}$ and $\left\langle\left\langle N_{\mathrm{a}}^{\mathrm{E}}(L)\right\rangle\right\rangle_{\mathrm{smpl}}$, which denote the numbers of non-zero coefficients before and after operating the random choice matrix $M_{\left\{\eta^{(L)}\right\}}$ to the state $\hat{H} M_{\left\{\eta^{(L-1)}\right\}} \cdots \hat{H} M_{\left\{\eta^{(1)}\right\}}\left|\psi_{\mathrm{E}}\right\rangle$. For comparison, these Tables also contain the results obtained by the SSS method. The value of the parameter $\epsilon$ in the SSS method is adjusted to be 0.06 so that $\left\langle\left\langle N_{\mathrm{b}}^{\mathrm{E}}(L)\right\rangle\right\rangle_{\text {smpl }}$ nearly equals to that in the RSSS method. In fact, Table II shows that $\left\langle\left\langle N_{\mathrm{b}}^{\mathrm{E}}(L)\right\rangle_{\mathrm{smpl}} \sim O\left(5 \times 10^{4}\right)\right.$ for $L \geq 6$ in the SSS method, which is comparable with $\left\langle\left\langle N_{\mathrm{b}}^{\mathrm{E}}(L)\right\rangle\right\rangle_{\mathrm{smpl}} \sim O\left(6 \times 10^{4}\right)$ for $L \geq 3$ in the RSSS method. We see that the RSSS results in Table I fairly reproduce the exact values and that, as is expected, errors are less than those in the SSS results. Although $\operatorname{Er}(L)$ is smaller in the SSS method when $L \leq 3$ due to the smaller value of $\epsilon$, it becomes smaller in the RSSS method for $L \geq 4$. The difference between errors in both methods grows as $L$ increases. The RSSS method therefore surely enables us to obtain meaningful results for larger values of $L$ compared to the SSS method.

Now we turn to the larger size system of 36 sites. For our purpose to evaluate the energy eigenvalue based on the power method, we first have to find an approximate state for this system. Here we use the restructuring techniques ${ }^{12)}$ to form fundamental basis states where three spins of each of non-overlapping triangles are diagonalized. Since neither simple way employed in the previous work $^{1,2)}$ could not give us a good approximate state, we combine them. Referring to the discussions in ref. 13 we start from a trial state and repeat the following procedure until the change of the obtained eigenvalue becomes negligibly small.

( a ) Operate the Hamiltonian to the trial state repeatedly as long as the number of the basis states is acceptable to our computer memory resources. Then a truncated vector space is formed.

( $b$ ) Calculate the lowest energy eigenvalue in the truncated vector space by means of the Lanczos method.

( c ) Drop small coefficients of the obtained eigenstate. Use it as the next trial state. Our best approximate state $\left|\psi_{\mathrm{A}}\right\rangle$ obtained from the above procedure is composed of 12,281,253 
non-zero components. The expectation value of $\hat{H}$ is $\left\langle\psi_{\mathrm{A}}|\hat{H}| \psi_{\mathrm{A}}\right\rangle=-18.418 .{ }^{14)}$ Using this $\left|\psi_{\mathrm{A}}\right\rangle$ we calculate the expectation values. Let $Q_{\mathrm{A}}(L) \equiv\left\langle\psi_{\mathrm{A}}\left|\hat{Q}^{L}\right| \psi_{\mathrm{A}}\right\rangle$, where $\hat{Q} \equiv 5 \hat{I}-\hat{H}$ with the identity operator $\hat{I}$. Since our method is based on the power method, we use $\hat{Q}^{L}$ instead of $\hat{H}^{L}$ in order to suppress excited states. Results on $\left\langle\left\langle Q_{\mathrm{A}\{\eta\}}(L)\right\rangle_{\mathrm{smpl}}\right.$ as well as $\left\langle\left\langle N_{\mathrm{b}}^{\mathrm{A}}(L)\right\rangle\right\rangle_{\mathrm{smpl}}$ and $\left\langle\left\langle N_{\mathrm{a}}^{\mathrm{A}}(L)\right\rangle\right\rangle_{\mathrm{smpl}}$ with $\epsilon=0.016$ and $n_{\mathrm{smpl}}=10^{3}$ are presented in Table III up to $L=12$. Here the maximum value of $L$ is determined under a condition that the square root of the variance, $\sqrt{\rho_{\{\eta\}}^{2}(L)}$, is less than the expectation value. The maximum program size is about one Gbytes and the CPU time is about one hour for one sampling by a Pentium 4 machine. The results in Table III show that the RSSS method is effective, not only for $\left|\psi_{\mathrm{E}}\right\rangle$ of the 21-site system but also for $\left|\psi_{\mathrm{A}}\right\rangle$ of the 36-site one, to suppress a rapid increase of the variance for large $L$. For example, the variance $\rho_{\{\eta\}}^{2}(5)$ in the SSS method is $9.3 \times 10^{13}$, which is about 600 times as large as the RSSS result $\rho_{\{\eta\}}^{2}(5)=1.6 \times 10^{11}$. We also see in Table III that the number of non-zero coefficients drastically reduces by the random choice matrix, $\left\langle\left\langle N_{\mathrm{a}}^{\mathrm{A}}(L)\right\rangle\right\rangle_{\mathrm{smpl}} \ll\left\langle\left\langle N_{\mathrm{b}}^{\mathrm{A}}(L)\right\rangle\right\rangle_{\mathrm{smpl}}$ for each $L$, and it increases very slowly as $L$ grows. In Figure 1 we plot the ratio $\left\langle\left\langle Q_{\mathrm{A}\{\eta\}}(L)\right\rangle\right\rangle_{\text {smpl }} /\left\langle\left\langle Q_{\mathrm{A}\{\eta\}}(L-1)\right\rangle\right\rangle_{\text {smpl }}$ $\left(\left\langle\left\langle Q_{\mathrm{A}\{\eta\}}(0)\right\rangle\right\rangle_{\mathrm{smpl}} \equiv 1\right)$, which should give the exact value $5-E$ in the large $L$ limit. We observe that the ratio increases for the data up to $L=10$. This increase suggests that the power method works. For $L=11$ and 12 the errors are too large to see this tendency. We therefore exclude $\left\langle\left\langle Q_{\mathrm{A}\{\eta\}}(11)\right\rangle\right\rangle_{\mathrm{smpl}}$ and $\left\langle\left\langle Q_{\mathrm{A}\{\eta\}}(12)\right\rangle\right\rangle_{\mathrm{smpl}}$ in the following analysis.

In order to estimate the energy eigenvalue from these expectation values we use the same fitting form as was introduced in the previous work, ${ }^{1)}$

$$
\left\langle\psi_{\mathrm{A}}\left|\hat{Q}^{L}\right| \psi_{\mathrm{A}}\right\rangle=Q^{L}\left(q_{0}+\frac{q_{1}}{L+\alpha+1}\right) \equiv F\left(L, Q, q_{0}, q_{1}, \alpha\right),
$$

$q_{0}, q_{1}, \alpha$ being free parameters which are to be determined together with $Q$ by the fit. Changing these four parameters under two constraints $q_{0}+q_{1} /(\alpha+1)=\left\langle\psi_{\mathrm{A}} \mid \psi_{\mathrm{A}}\right\rangle=1$ and $Q\left\{q_{0}+q_{1} /(\alpha+2)\right\}=$ $\left\langle\psi_{\mathrm{A}}|\hat{Q}| \psi_{\mathrm{A}}\right\rangle=-18.418$, we look for the minimum of the difference $D$,

$$
D \equiv \sum_{L=2}^{L_{\max }}\left[1-\frac{\left\langle\left\langle Q_{\mathrm{A}\{\eta\}}(L)\right\rangle\right\rangle_{\mathrm{smpl}}}{F\left(L, Q, q_{0}, q_{1}, \alpha\right)}\right]^{2},
$$

with $L_{\max }=10$. We accept the values of the parameters if $D$ is less than the sum of the relative errors

$$
\sum_{L=2}^{L_{\max }}\left[\frac{E r(L)}{\left\langle\left\langle Q_{\mathrm{A}\{\eta\}}(L)\right\rangle\right\rangle_{\mathrm{smpl}}}\right]^{2}=2.2 \times 10^{-3} .
$$

The result of this fit is $E_{\mathrm{fit}}=5-Q_{\mathrm{fit}}=-20.50 \pm \begin{aligned} & 1.37 \\ & 0.12\end{aligned}$. The function $F\left(L, Q_{\mathrm{fit}}, q_{\text {ofit }}, q_{1 \mathrm{fit}}, \alpha_{\mathrm{fit}}\right)$ with fitted parameters to minimize $D$ is plotted with a solid line in Fig. 1. 


\section{Further Calculations}

In this section we add several results for the 36-site system. In the previous section we obtained, starting from an approximate state $\left|\psi_{\mathrm{A}}\right\rangle$, the ground state energy of the 36-site system which is in agreement with the known exact value. Statistical errors, however, are not satisfactorily small. One way to decrease the errors is to increase the number of samples, but it is quite time-consuming. In this section we employ another way, which is to improve the trial state by requesting the translational invariance. For this purpose we introduce a wave vector $\mathbf{k}$. Roughly every $N_{\mathrm{s}}$ basis states are linearly combined to form one new basis state for a definite value of $\mathbf{k}$, where $N_{\mathrm{s}}$ denotes the number of sites of the system. Requesting $\mathbf{k}=\mathbf{0}$ we construct an improved trial state $\left|\psi_{\mathrm{T}}\right\rangle$, whose number of new basis states amounts 13,911,394 and $\left\langle\psi_{\mathrm{T}}|\hat{H}| \psi_{\mathrm{T}}\right\rangle=-19.710$. Here we abandon the restructuring techniques in order to avoid too much complexity. Results up to $L=6$ from 140 samples with $\epsilon=0.02$ are shown in Table IV and Fig. 1. Using the same fitting form (24) we obtain $E_{\mathrm{fit}}=-20.04 \pm \frac{0.10}{0.06}$, whose statistical errors are less than $0.5 \%$. The number of nonzero coefficients before (after) operating the 6 -th random choice matrix is $\left\langle\left\langle N_{\mathrm{b}}^{\mathrm{T}}(6)\right\rangle_{\mathrm{smpl}} \sim 3.0 \times 10^{7}\right.$ $\left(\left\langle\left\langle N_{\mathrm{a}}^{\mathrm{T}}(6)\right\rangle\right\rangle_{\mathrm{smpl}} \sim 7.9 \times 10^{5}\right)$.

The lowest energy eigenvalues for excited states with fixed values of $S_{z}$, the $z$-component of the total spin, are also calculable in similar manners. Our results for the $S_{z}=1$ homogeneous $(\mathbf{k}=\mathbf{0})$ state are presented in Table V. Here $\epsilon=0.02$ and $n_{\text {smpl }}=88$ for $L \leq 5$, while $\epsilon=0.03$ and $n_{\mathrm{smpl}}=180$ for $L \geq 6$. We start from a trial state $\left|\psi_{\mathrm{T}}\left(S_{z}=1\right)\right\rangle$, for which $\left\langle\psi_{\mathrm{T}}\left(S_{z}=1\right)|\hat{H}| \psi_{\mathrm{T}}\left(S_{z}=1\right)\right\rangle=-18.813$. Up to $L=5$ we, as usual, calculate inner products of $\left\langle\psi_{\mathrm{T}}\left(S_{z}=1\right)\right|$ and $\hat{Q} M_{\left\{\eta^{(L)}\right\}} \cdots \hat{Q} M_{\left\{\eta^{(1)}\right\}}\left|\psi_{\mathrm{T}}\left(S_{z}=1\right)\right\rangle$. For $L \geq 6$, on the other hand, we measure inner products between independently generated $\left\langle\psi_{\mathrm{T}}\left(S_{z}=1\right)\right| M_{\left\{\eta^{\prime(1)}\right\}} \hat{Q} \cdots M_{\left\{\eta^{\prime(L-5)\}}\right.} \hat{Q}$ and $\hat{Q} M_{\left\{\eta^{(5)}\right\}} \cdots \hat{Q} M_{\left\{\eta^{(1)}\right\}}\left|\psi_{\mathrm{T}}\left(S_{z}=1\right)\right\rangle$ in order to save memory resources. This measurement is allowed because $\left\langle\left\langle\left\langle\psi\left|M_{\left\{\eta^{\prime}(1)\right\}} \hat{Q} \cdots M_{\left\{\eta^{\prime}(L-5)\right\}} \hat{Q} \cdot \hat{Q} M_{\left\{\eta^{(5)}\right\}} \cdots \hat{Q} M_{\left\{\eta^{(1)}\right\}}\right| \psi\right\rangle\right\rangle=\left\langle\left\langle\left\langle\psi\left|\hat{Q}^{L}\right| \psi\right\rangle\right\rangle\right\rangle\right.$ holds. ${ }^{15)}$ Based on the assumption (24), we obtain $E_{\text {fit }}=-19.21 \pm{ }_{0.10}^{0.27}$ from data in Table V.

Finally, in order to demonstrate that we can extract physical properties of the ground state with this method, we report results on the static structure factor $F(\mathbf{k})$ defined by

$$
F(\mathbf{k}) \equiv\left\langle\psi_{\mathrm{E}}\left|\mathbf{S}_{-\mathbf{k}} \cdot \mathbf{S}_{\mathbf{k}}\right| \psi_{\mathrm{E}}\right\rangle, \quad \mathbf{S}_{\mathbf{k}} \equiv \frac{1}{2 \sqrt{N_{\mathrm{s}}}} \sum_{j} \boldsymbol{\sigma}_{j} e^{-i \mathbf{k} \mathbf{r}_{j}}
$$

where $j$ runs over all site of the lattice and $\mathbf{r}_{j}$ denotes the position vector for site $j$. In numerical study we define

$$
F_{m}(\mathbf{k}) \equiv \frac{\left\langle\psi_{\mathrm{T}}\left|\hat{Q}^{m} \mathbf{S}_{-\mathbf{k}} \cdot \mathbf{S}_{\mathbf{k}} \hat{Q}^{m}\right| \psi_{\mathrm{T}}\right\rangle}{\left\langle\psi_{\mathrm{T}}\left|\hat{Q}^{2 m}\right| \psi_{\mathrm{T}}\right\rangle}
$$


which gives $F(\mathbf{k})$ in the large $m$ limit,

$$
\lim _{m \rightarrow \infty} F_{m}(\mathbf{k})=F(\mathbf{k}) .
$$

Then we evaluate $F_{m}(\mathbf{k})$ from measurements of

$$
\frac{\left\langle\left\langle\left\langle\psi_{\mathrm{T}}\left|\hat{Q} M_{\left\{\eta^{(2 m-2)}\right\}} \cdots \hat{Q} M_{\left\{\eta^{(m)}\right\}} \mathbf{S}_{-\mathbf{k}} \cdot \mathbf{S}_{\mathbf{k}} \hat{Q} M_{\left\{\eta^{(m-1)}\right\}} \cdots \hat{Q} M_{\left\{\eta^{(1)}\right\}}\right| \psi_{\mathrm{T}}\right\rangle\right\rangle_{\mathrm{smpl}}\right.}{\left\langle\left\langle\left\langle\psi_{\mathrm{T}}\left|\hat{Q} M_{\left\{\eta^{(2 m-2)}\right\}} \cdots \hat{Q} M_{\left\{\eta^{(m)}\right\}} \hat{Q} M_{\left\{\eta^{(m-1)}\right\}} \cdots \hat{Q} M_{\left\{\eta^{(1)}\right\}}\right| \psi_{\mathrm{T}}\right\rangle\right\rangle_{\mathrm{smpl}}\right.} .
$$

Our results for $\epsilon=0.03, n_{\text {smpl }}=8$ and $m=6$ are shown in Fig. 2. Here we do not need many samples because we allow maximally one per cent of the statistical errors for $F_{m}(\mathbf{k})$. They are in good agreement with the data from ref. 7 except for those with $k / k_{0}=1$. When $k=k_{0}$ we can obtain a better value from the squared sublattice magnetization $\mathcal{M}^{2}$, using the relation ${ }^{16)}$ $2 N_{\mathrm{s}} F\left(\mathbf{k}_{0}\right)=9 \mathcal{M}^{2}$. The reason for this better value is that in measurement of $\mathcal{M}^{2}$ one does not suffer from heavy cancellations. We find this value is much closer to the datum given in ref. 7 .

\section{Summary and Discussions}

In this paper we developed the recursive stochastic state selection (RSSS) method, which is derived from the stochastic state selection (SSS) method. ${ }^{1)}$ As the word "recursive" indicates, on-off probability functions to generate the random choice matrix are recurrently determined so that they reflect the newest intermediate state at each step of the RSSS procedure. The merit of this modification is that variances for expectation values with high powers of the Hamiltonian grow slowly compared with those in the SSS method.

As a result of less variances we can now evaluate the ground state energy for the triangular lattice Heisenberg spin-1/2 system by means of the stochastic selections. On a relatively small 21-site lattice we examined the RSSS method using the exact eigenstate. Results there show that

the variance $\rho_{\{\eta\}}^{2}(10)$ was reduced to $\sim 1 / 2000$ of the one in the SSS method. On a larger 36-site lattice we calculated the expectation values of $(5 \hat{I}-\hat{H})^{L}$ from two approximate states $\left|\psi_{\mathrm{A}}\right\rangle$ and $\left|\psi_{\mathrm{T}}\right\rangle$, where the latter realizes the wave vector $\mathbf{k}=\mathbf{0}$. We again emphasize that the numerical study of such high powers would not become possible without introducing the RSSS method. From obtained data with $\left|\psi_{\mathrm{T}}\right\rangle$ we estimated the energy of the ground state $E$ based on the extrapolation assumption. The result indicates $-20.10 \leq E \leq-19.94$, which is slightly higher than the known exact value -20.17344 . On the 36-site triangular lattice we also calculated expectation values on the lowest energy eigenvalue with the $S_{z}=1$ state. Further, we calculated the static structure factor, which is a typical measurement to obtain physical properties of the ground state. Our results fully ensure that not only energy eigenvalues but also the static structure factor is calculable in our method.

Let us finally comment on whether it is possible to study larger systems, the 48-site system for instance, by this method. In a simple estimation by a guess that the cost is proportional to 
the system size, we would need $2^{48} / 2^{36} \sim 4 \times 10^{3}$ times of computer resources compared to the the CPU time and the memory we used in our present study for the 36-site system. This factor is too large for us to easily overcome. We, however, show in this study that a better trial state with a fixed $\mathbf{k}$ can much reduce the cost of calculations. We hope, therefore, we can make a numerical study of larger triangular spin systems in the near future.

\section{Appendix: On-Off Probability Functions}

In this Appendix we consider a random choice matrix $M_{\{\eta\}} \equiv$ diag. $\left\{\eta_{1}, \eta_{2}, \cdots, \eta_{N_{\mathrm{V}}}\right\}$, where each random variable $\eta_{i}$ is generated by an on-off probability function defined with a parameter $\xi_{i}$ $(\geq 1)$,

$$
P_{i}(\eta) \equiv P\left(\eta ; \xi_{i}\right)=\frac{1}{\xi_{i}} \delta\left(\eta-\xi_{i}\right)+\left(1-\frac{1}{\xi_{i}}\right) \delta(\eta) .
$$

Our purpose is to discuss what values of $\{\xi\} \equiv\left\{\xi_{1}, \xi_{2}, \cdots, \xi_{N_{\mathrm{V}}}\right\}$ are favorable in order to decrease the variances of the expectation values. We will see that the definition of the on-off probability functions in the RSSS method is "best" in the sense shown below.

First, let $N_{i}$ denote the number of non-zero $\eta_{i}$, which is therefore $1(0)$ when $\eta_{i} \neq 0\left(\eta_{i}=0\right)$.

Following the discussion in ref. 1, we obtain for the statistical average of $N \equiv \sum N_{i}$ in the above $M_{\{\eta\}}$,

$$
\langle\langle N\rangle\rangle=\sum_{i=1}^{N_{\mathrm{V}}} \frac{1}{\xi_{i}}
$$

because

$$
\begin{aligned}
\left\langle\left\langle N_{i}\right\rangle\right\rangle & \equiv \int_{0}^{\infty} N_{i} P_{N_{i}}\left(N_{i}\right) d N_{i}=\frac{1}{\xi_{i}}, \\
P_{N_{i}}\left(N_{i}\right) & \equiv \frac{1}{\xi_{i}} \delta\left(N_{i}-1\right)+\left(1-\frac{1}{\xi_{i}}\right) \delta\left(N_{i}\right) .
\end{aligned}
$$

Next, multiplying $M_{\{\eta\}}$ to a normalized state vector $|\Psi\rangle$,

$$
|\Psi\rangle=\sum_{i=1}^{N_{\mathrm{V}}}|i\rangle c_{i}
$$

we obtain

$$
\begin{aligned}
M_{\{\eta\}}|\Psi\rangle & =\sum_{i}|i\rangle c_{i} \eta_{i}, \\
\left\langle\Psi\left|\left[M_{\{\eta\}}\right]^{2}\right| \Psi\right\rangle & =\sum_{i} c_{i}^{2} \eta_{i}^{2}=1+\sum_{i} c_{i}^{2}\left(\eta_{i}^{2}-1\right)
\end{aligned}
$$


where the normalization condition $\langle\Psi \mid \Psi\rangle=\sum c_{i}^{2}=1$ is used. Let us then introduce a state vector $|\chi\rangle g$ which represents the difference between $M_{\{\eta\}}|\Psi\rangle$ and $|\Psi\rangle$,

$$
|\chi\rangle g \equiv M_{\{\eta\}}|\Psi\rangle-|\Psi\rangle=\sum_{i}|i\rangle c_{i}\left(\eta_{i}-1\right)
$$

with a normalized $|\chi\rangle$. It should be emphasized here that $\langle\langle\langle\Phi \mid \chi\rangle g\rangle=0$ holds for any state $|\Phi\rangle \equiv \sum|i\rangle b_{i}$ because $\left\langle\left\langle\eta_{i}\right\rangle\right\rangle=1$. From (A.8) we see

$$
\left\langle\left\langle\left\langle\Psi\left[M_{\{\eta\}}\right]^{2} \mid \Psi\right\rangle\right\rangle=\left\langle\left\langle\langle\Psi \mid \Psi\rangle+2 g\langle\chi \mid \Psi\rangle+g^{2}\langle\chi \mid \chi\rangle\right\rangle\right\rangle=1+\left\langle\left\langle g^{2}\right\rangle\right\rangle .\right.
$$

Using

$$
\left\langle\left\langle\eta_{i}^{2}\right\rangle\right\rangle \equiv \int_{0}^{\infty} \eta_{i}^{2} P_{i}\left(\eta_{i}\right) d \eta_{i}=\xi_{i}
$$

we obtain from $(\mathrm{A} \cdot 7)$ and $(\mathrm{A} \cdot 9)$,

$$
\left\langle\left\langle g^{2}\right\rangle\right\rangle=\left\langle\left\langle\sum_{i} c_{i}^{2}\left(\eta_{i}^{2}-1\right)\right\rangle\right\rangle=\sum_{i} c_{i}^{2}\left(\left\langle\left\langle\eta_{i}^{2}\right\rangle\right\rangle-1\right)=\sum_{i} c_{i}^{2}\left(\xi_{i}-1\right)
$$

Is it possible to find any $\{\xi\}$ which makes both of $\langle\langle N\rangle\rangle$ and $\left\langle\left\langle g^{2}\right\rangle\right\rangle$ small? From (A.2) and (A.11) we learn that we cannot lessen $\left\langle\left\langle g^{2}\right\rangle\right\rangle$ without increasing $\langle\langle N\rangle\rangle$. Let us consider, then, to minimize a quantity

$$
\epsilon^{2}\langle\langle N\rangle\rangle+\left\langle\left\langle g^{2}\right\rangle\right\rangle=\sum_{i=1}^{N_{\mathrm{V}}}\left\{\frac{\epsilon^{2}}{\xi_{i}}+c_{i}^{2}\left(\xi_{i}-1\right)\right\} \equiv S\left(\xi_{1}, \xi_{2}, \cdots, \xi_{N_{\mathrm{V}}}\right),
$$

with a positive constant $\epsilon$ in ranges $\xi_{i} \geq 1$ for all $i$. Since $\partial S / \partial \xi_{i}=c_{i}^{2}-\epsilon^{2} / \xi_{i}^{2}$, we find

- $\partial S / \partial \xi_{i}=0$ at $\xi_{i}=\epsilon /\left|c_{i}\right|(>1)$ if $0<\left|c_{i}\right|<\epsilon$.

- $\partial S / \partial \xi_{i}>0$ for $\xi_{i} \geq 1$ when $\left|c_{i}\right|>\epsilon$. In this case $\xi_{i}=1$ gives the minimum of $\epsilon^{2} / \xi_{i}+c_{i}^{2}\left(\xi_{i}-1\right)$.

- $\partial S / \partial \xi_{i}<0$ if $c_{i}=0$. In this case $\epsilon^{2} / \xi_{i}+c_{i}^{2}\left(\xi_{i}-1\right)=\epsilon^{2} / \xi_{i} \rightarrow 0$ when $\xi_{i} \rightarrow \infty$.

Therefore the minimum value of $S\left(\xi_{1}, \xi_{2}, \cdots, \xi_{N_{\mathrm{V}}}\right)$ is realized by

$$
\xi_{i}= \begin{cases}\max \left(1, \epsilon /\left|c_{i}\right|\right) & \left(c_{i} \neq 0\right) \\ \infty & \left(c_{i}=0\right)\end{cases}
$$

To summarize this Appendix, we find the "best" choice of $\{\xi\}$ in $(A \cdot 1)$, by which the quantity $\epsilon^{2}\langle\langle N\rangle\rangle+\left\langle\left\langle g^{2}\right\rangle\right\rangle$ in $(\mathrm{A} \cdot 12)$ is minimized. 
1) T. Munehisa and Y. Munehisa: J. Phys. Soc. Jpn. 72 (2003) 2759.

2) T. Munehisa and Y. Munehisa: J. Phys. Soc. Jpn. 73 (2004) 340.

3) T. Oguchi, H. Nishimori and Y. Taguchi: J. Phys. Soc. Jpn 55 (1986) 323.

4) H. Nishimori and H. Nakanishi: J. Phys. Soc. Jpn 57 (1988) 626.

5) P. W. Leung and K. J. Runge: Phys. Rev. B47 (1993) 5861.

6) P. Sindzingre, P. Lecheminant and C. Lhuillier: Phys. Rev. B50 (1994) 3108.

7) B. Bernu, P. Lecheminant, C. Lhuillier and L. Pierre: Phys. Rev. B50 (1994) 10048.

8) L. Capriotti, A. E. Trumper and S. Sorella: Phys. Rev. Lett. 82 (1999) 3899.

9) D.J. Farnell, R.Bishop and K.Gernoth: Phys. Rev. B64 (2001) 172409.

10) In the SSS method we first defined $E_{\{\eta\}}(L)$ as

$$
E_{\{\eta\}}(L) \equiv\left\langle\psi\left|M_{\left\{\eta^{(L+1)}\right\}} \hat{H} M_{\left\{\eta^{(L)}\right\}} \hat{H} M_{\left\{\eta^{(L-1)}\right\}} \cdots \hat{H} M_{\left\{\eta^{(1)}\right\}}\right| \psi\right\rangle,
$$

but in numerical calculations we often use

$$
E_{\{\eta\}}(L) \equiv\left\langle\psi\left|\hat{H} M_{\left\{\eta^{(L)}\right\}} \hat{H} M_{\left\{\eta^{(L-1)}\right\}} \cdots \hat{H} M_{\left\{\eta^{(1)}\right\}}\right| \psi\right\rangle
$$

in order to decrease statistical errors.

11) For the 21-site lattice these directions are along vectors $\boldsymbol{T}_{1}=4 \boldsymbol{u}_{1}+\boldsymbol{u}_{2}$ and $\boldsymbol{T}_{2}=-\boldsymbol{u}_{1}+5 \boldsymbol{u}_{2}$, where $\boldsymbol{u}_{1}=(1,0)$ and $\boldsymbol{u}_{2}=(1 / 2, \sqrt{3} / 2)$ are unit vectors for the triangular lattice in the $x-y$ plain. For the 36 -site lattice they are along $\boldsymbol{T}_{1}=6 \boldsymbol{u}_{1}$ and $\boldsymbol{T}_{2}=6 \boldsymbol{u}_{2}$.

12) T. Munehisa and Y. Munehisa: Phys. Rev. B49 (1994) 3347; Prog. Theor. Phys. 92 (1994) 309; Prog. Theor. Phys. 93 (1995) 251; Prog. Theor. Phys. 96 (1996) 59.

13) J. Riera and E. Dagotto: Phys. Rev. B48 (1993) 9515.

14) In the normal representation the state on each site of the lattice is represented by the $z$ component of the spin sitting on the site. In this representation we obtain $\left\langle\psi_{\mathrm{A}}^{\prime}|\hat{H}| \psi_{\mathrm{A}}^{\prime}\right\rangle=-17.6398$ for our best approximate state $\left|\psi_{\mathrm{A}}^{\prime}\right\rangle$.

15) For example, using (19) we see that

$$
\begin{aligned}
\hat{Q} M_{\left\{\eta^{(1)}\right\}}|\psi\rangle & =\hat{Q}|\psi\rangle+\hat{Q}|\chi\rangle g, \\
\hat{Q} M_{\left\{\eta^{\prime}(1)\right\}}|\psi\rangle & =\hat{Q}|\psi\rangle+\hat{Q}\left|\chi^{\prime}\right\rangle g^{\prime},
\end{aligned}
$$

and then

$$
\begin{aligned}
& \left\langle\left\langle\left\langle\psi\left|M_{\left\{\eta^{\prime}(1)\right\}} \hat{Q} \cdot \hat{Q} M_{\left\{\eta^{(1)}\right\}}\right| \psi\right\rangle\right\rangle\right\rangle \\
= & \left\langle\left\langle\left\langle\psi\left|\hat{Q}^{2}\right| \psi\right\rangle\right\rangle+\left\langle\left\langle g^{\prime}\left\langle\chi^{\prime}\left|\hat{Q}^{2}\right| \psi\right\rangle\right\rangle+\left\langle\left\langle\left\langle\psi\left|\hat{Q}^{2}\right| \chi\right\rangle g\right\rangle\right\rangle+\left\langle\left\langle g^{\prime}\left\langle\chi^{\prime}\left|\hat{Q}^{2}\right| \chi\right\rangle g\right\rangle\right\rangle\right.\right. \\
= & \left\langle\left\langle\left\langle\psi\left|\hat{Q}^{2}\right| \psi\right\rangle\right\rangle .\right.
\end{aligned}
$$

16) This is an identity for systems with the total $\operatorname{spin} \mathbf{S}=\mathbf{0} .^{7)}$ 


\begin{tabular}{|c|c|c|c|c|c|c|}
\hline \multirow[b]{2}{*}{$L$} & \multirow{2}{*}{\multicolumn{2}{|c|}{$E_{\mathrm{E}}(L)$}} & \multicolumn{2}{|l|}{ RSSS } & \multicolumn{2}{|l|}{ SSS } \\
\hline & & & & $\left\langle\left\langle E_{\mathrm{E}\{\eta\}}\right.\right.$ & L) $\rangle_{\mathrm{smpl}}$ & \\
\hline 1 & -0.117809 & $\times 10^{2}$ & $-(0.117787 \pm 0.000072)$ & $\times 10^{2}$ & $-(0.117796 \pm 0.000050)$ & $\times 10^{2}$ \\
\hline 2 & 0.138790 & $\times 10^{3}$ & $(0.13885 \pm 0.00015)$ & $\times 10^{3}$ & $(0.138760 \pm 0.000098)$ & $\times 10^{3}$ \\
\hline 3 & -0.163507 & $\times 10^{4}$ & $-(0.16362 \pm 0.00027)$ & $\times 10^{4}$ & $-(0.16343 \pm 0.00020)$ & $\times 10^{4}$ \\
\hline 4 & 0.192626 & $\times 10^{5}$ & $(0.19286 \pm 0.00044)$ & $\times 10^{5}$ & $(0.19244 \pm 0.00067)$ & $\times 10^{5}$ \\
\hline 5 & -0.226931 & $\times 10^{6}$ & $-(0.22710 \pm 0.00070)$ & $\times 10^{6}$ & $-(0.2270 \pm 0.0030)$ & $\times 10^{6}$ \\
\hline 6 & 0.267346 & $\times 10^{7}$ & $(0.2671 \pm 0.0011)$ & $\times 10^{7}$ & $(0.269 \pm 0.015)$ & $\times 10^{7}$ \\
\hline 7 & -0.314958 & $\times 10^{8}$ & $-(0.3152 \pm 0.0018)$ & $\times 10^{8}$ & $-(0.286 \pm 0.049)$ & $\times 10^{8}$ \\
\hline 8 & 0.371049 & $\times 10^{9}$ & $(0.3708 \pm 0.0030)$ & $\times 10^{9}$ & $(0.24 \pm 0.17)$ & $\times 10^{9}$ \\
\hline 9 & -0.437129 & $\times 10^{10}$ & $-(0.4357 \pm 0.0050)$ & $\times 10^{10}$ & $-(0.39 \pm 0.17)$ & $\times 10^{10}$ \\
\hline 10 & 0.514978 & $\times 10^{11}$ & $(0.5170 \pm 0.0085)$ & $\times 10^{11}$ & $(0.30 \pm 0.41)$ & $\times 10^{11}$ \\
\hline 11 & -0.606691 & $\times 10^{12}$ & $-(0.607 \pm 0.014)$ & $\times 10^{12}$ & - & \\
\hline 12 & 0.714737 & $\times 10^{13}$ & $(0.709 \pm 0.025)$ & $\times 10^{13}$ & - & \\
\hline 13 & -0.842025 & $\times 10^{14}$ & $-(0.850 \pm 0.042)$ & $\times 10^{14}$ & - & \\
\hline 14 & 0.991982 & $\times 10^{15}$ & $(1.005 \pm 0.072)$ & $\times 10^{15}$ & - & \\
\hline 15 & -0.116865 & $\times 10^{17}$ & $-(0.122 \pm 0.013)$ & $\times 10^{17}$ & - & \\
\hline
\end{tabular}

Table I. Results on $\left\langle\left\langle E_{\mathrm{E}\{\eta\}}(L)\right\rangle\right\rangle_{\mathrm{smpl}}(L=1,2, \cdots, 15)$ obtained for the 21-site triangular lattice by the RSSS method with $\epsilon=0.1$ and the SSS method with $\epsilon=0.06$. The number of samples is $10^{4}$. Exact values of $E_{\mathrm{E}}(L)$ are also presented for comparison. 


\begin{tabular}{|c|c|c|c|c|}
\hline \multirow[b]{2}{*}{$L$} & \multicolumn{2}{|c|}{ RSSS } & \multicolumn{2}{|c|}{ SSS } \\
\hline & $\left\langle\left\langle N_{\mathrm{b}}^{\mathrm{E}}(L)\right\rangle\right\rangle_{\mathrm{smpl}}$ & $\left\langle\left\langle N_{\mathrm{a}}^{\mathrm{E}}(L)\right\rangle\right\rangle_{\mathrm{smpl}}$ & $\left\langle\left\langle N_{\mathrm{b}}^{\mathrm{E}}(L)\right\rangle\right\rangle_{\mathrm{smpl}}$ & $\left\langle\left\langle N_{\mathrm{a}}^{\mathrm{E}}(L)\right\rangle\right\rangle_{\mathrm{smpl}}$ \\
\hline 1 & 352716.0 & 2659.3 & 352716.0 & 4432.2 \\
\hline 2 & 74254.9 & 2038.3 & 108023.1 & 2603.0 \\
\hline 3 & 60343.5 & 1923.6 & 70882.0 & 2197.7 \\
\hline 4 & 57531.4 & 1920.4 & 60150.5 & 2048.7 \\
\hline 5 & 57365.5 & 1946.4 & 56182.7 & 1984.9 \\
\hline 6 & 57819.9 & 1975.7 & 54473.2 & 1955.8 \\
\hline 7 & 58330.5 & 2000.4 & 53687.7 & 1941.7 \\
\hline 8 & 58775.9 & 2020.2 & 53304.5 & 1934.3 \\
\hline 9 & 59672.5 & 2033.5 & 53102.0 & 1930.7 \\
\hline 10 & 60088.4 & 2043.6 & 53003.4 & 1929.1 \\
\hline 11 & 60185.8 & 2050.4 & - & - \\
\hline 12 & 60143.4 & 2053.9 & - & - \\
\hline 13 & 60179.8 & 2057.8 & - & - \\
\hline 14 & 60624.2 & 2059.9 & - & - \\
\hline 15 & 60691.2 & 2061.4 & - & - \\
\hline
\end{tabular}

Table II. Numbers of non-zero coefficients before $\left(\left\langle\left\langle N_{\mathrm{b}}^{\mathrm{E}}(L)\right\rangle\right\rangle_{\mathrm{smpl}}\right)$ and after $\left(\left\langle\left\langle N_{\mathrm{a}}^{\mathrm{E}}(L)\right\rangle\right\rangle_{\mathrm{smpl}}\right)$ operating the random choice matrix $M_{\left\{\eta^{(L)}\right\}}$ to the state $\hat{H} M_{\left\{\eta^{(L-1)}\right\}} \cdots \hat{H} M_{\left\{\eta^{(1)}\right\}}\left|\psi_{E}\right\rangle$ for the 21-site triangular lattice obtained from $10^{4}$ samples with $\epsilon=0.1$ (RSSS) or $\epsilon=0.06$ (SSS). 


\begin{tabular}{|c|c|c|c|c|}
\hline$L$ & $\left\langle\left\langle Q_{\mathrm{A}\{\eta\}}(L)\right\rangle\right\rangle_{\mathrm{smpl}}$ & & $\left\langle\left\langle N_{\mathrm{b}}^{\mathrm{A}}(L)\right\rangle\right\rangle_{\mathrm{smpl}}$ & $\left\langle\left\langle N_{\mathrm{a}}^{\mathrm{A}}(L)\right\rangle\right\rangle_{\mathrm{smpl}}$ \\
\hline 1 & $(0.234137 \pm 0.000077)$ & $\times 10^{2}$ & 12281253.0 & 91378.8 \\
\hline 2 & $(0.55286 \pm 0.00045)$ & $\times 10^{3}$ & 21738077.0 & 69452.6 \\
\hline 3 & $(0.13114 \pm 0.00019)$ & $\times 10^{5}$ & 19123511.3 & 69429.2 \\
\hline 4 & $(0.31260 \pm 0.00071)$ & $\times 10^{6}$ & 19821436.4 & 74755.3 \\
\hline 5 & $(0.7469 \pm 0.0025)$ & $\times 10^{7}$ & 21556264.4 & 81933.1 \\
\hline 6 & $(0.17941 \pm 0.00093)$ & $\times 10^{9}$ & 23674697.9 & 89782.3 \\
\hline 7 & $(0.4296 \pm 0.0035)$ & $\times 10^{10}$ & 25924492.7 & 97767.5 \\
\hline 8 & $(0.1028 \pm 0.0014)$ & $\times 10^{12}$ & 28180669.0 & 105616.8 \\
\hline 9 & $(0.2507 \pm 0.0055)$ & $\times 10^{13}$ & 30379138.4 & 113156.1 \\
\hline 10 & $(0.629 \pm 0.024)$ & $\times 10^{14}$ & 32478107.9 & 120316.8 \\
\hline 11 & $(0.153 \pm 0.010)$ & $\times 10^{16}$ & 34462103.9 & 127006.7 \\
\hline 12 & $(0.355 \pm 0.042)$ & $\times 10^{17}$ & 36309064.0 & 133239.7 \\
\hline
\end{tabular}

Table III. Results on $\left\langle\left\langle Q_{\mathrm{A}\{\eta\}}(L)\right\rangle\right\rangle_{\mathrm{smpl}},\left\langle\left\langle N_{\mathrm{b}}^{\mathrm{A}}(L)\right\rangle\right\rangle_{\mathrm{smpl}}$ and $\left\langle\left\langle N_{\mathrm{a}}^{\mathrm{A}}(L)\right\rangle\right\rangle_{\mathrm{smpl}}(L=1,2, \cdots, 12)$, obtained for the 36 -site restructured triangular lattice by the RSSS method with $\epsilon=0.016$, where $Q_{\mathrm{A}}(L)=\left\langle\psi_{\mathrm{A}}\left|(5 \hat{I}-\hat{H})^{L}\right| \psi_{\mathrm{A}}\right\rangle$ with the identity operator $\hat{I}$. The number of samples is $10^{3}$. 


\begin{tabular}{rrrrr}
\hline$L$ & $\left\langle\left\langle Q_{\mathrm{T}\{\eta\}}(L)\right\rangle\right\rangle_{\mathrm{smpl}}$ & & $\left\langle\left\langle N_{\mathrm{b}}^{\mathrm{T}}(L)\right\rangle\right\rangle_{\mathrm{smpl}}$ & $\left\langle\left\langle N_{\mathrm{a}}^{\mathrm{T}}(L)\right\rangle\right\rangle_{\mathrm{smpl}}$ \\
\hline 1 & $(0.247073 \pm 0.000045)$ & $\times 10^{2}$ & 13911394.0 & 398861.8 \\
\hline 2 & $(0.612899 \pm 0.000193)$ & $\times 10^{3}$ & 13513630.8 & 437278.8 \\
\hline 3 & $(0.152364 \pm 0.000063)$ & $\times 10^{5}$ & 16928438.7 & 518694.1 \\
\hline 4 & $(0.37929 \pm 0.00018)$ & $\times 10^{6}$ & 21395583.2 & 610462.3 \\
\hline 5 & $(0.94546 \pm 0.00057)$ & $\times 10^{7}$ & 25728449.4 & 701952.5 \\
\hline 6 & $(0.23594 \pm 0.00017)$ & $\times 10^{9}$ & 30450059.3 & 787238.1 \\
\hline
\end{tabular}

Table IV. Results on $\left\langle\left\langle Q_{\mathrm{T}\{\eta\}}(L)\right\rangle\right\rangle_{\mathrm{smpl}},\left\langle\left\langle N_{\mathrm{b}}^{\mathrm{T}}(L)\right\rangle\right\rangle_{\mathrm{smpl}}$ and $\left\langle\left\langle N_{\mathrm{a}}^{\mathrm{T}}(L)\right\rangle\right\rangle_{\mathrm{smpl}}(L=1,2, \cdots, 12)$ obtained for the 36 -site triangular lattice by the RSSS method with $\epsilon=0.02$, where a trial state $\left|\psi_{\mathrm{T}}\right\rangle$ is used instead of $\left|\psi_{\mathrm{A}}\right\rangle$. The number of samples is 140 .

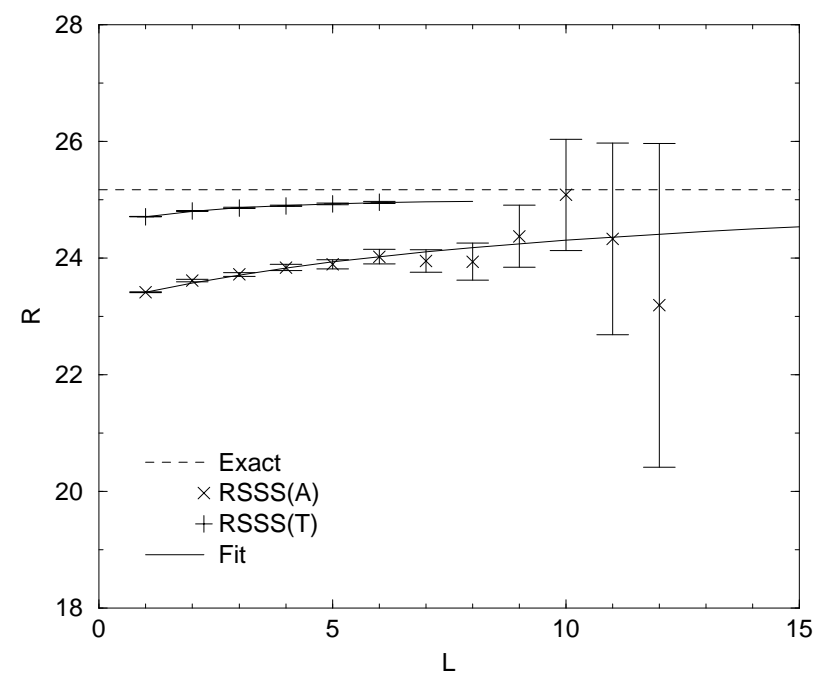

Fig. 1. Ratios $R_{\mathrm{A}} \equiv\left\langle\left\langle Q_{\mathrm{A}\{\eta\}}(L)\right\rangle\right\rangle_{\mathrm{smpl}} /\left\langle\left\langle Q_{\mathrm{A}\{\eta\}}(L-1)\right\rangle\right\rangle_{\mathrm{smpl}}$ (crosses) and $R_{\mathrm{T}} \equiv\left\langle\left\langle Q_{\mathrm{T}\{\eta\}}(L)\right\rangle\right\rangle_{\mathrm{smpl}} /\left\langle\left\langle Q_{\mathrm{T}\{\eta\}}(L-1)\right\rangle\right\rangle_{\mathrm{smpl}}$ (pluses) for the Heisenberg spin model on the 36-site triangular lattice. Values of $R_{\mathrm{A}}$ are obtained from the results in Table III and $\left\langle\left\langle Q_{\mathrm{A}\{\eta\}}(0)\right\rangle\right\rangle_{\mathrm{smpl}} \equiv 1$. Values of $R_{\mathrm{T}}$ are obtained from the results in Table IV and $\left\langle\left\langle Q_{\mathrm{T}\{\eta\}}(0)\right\rangle\right\rangle_{\mathrm{smpl}} \equiv 1$. The dashed line indicates the exact eigenvalue of $Q=5-E .^{5,7,8)}$ The solid lines present fitted values of (24). 


\begin{tabular}{|c|c|c|c|c|}
\hline$L$ & $\left\langle\left\langle Q_{\mathrm{T}\{\eta\}}(L)\right\rangle\right\rangle_{\mathrm{smpl}}$ & & $\left\langle\left\langle N_{\mathrm{b}}^{\mathrm{T}}(L)\right\rangle\right\rangle_{\mathrm{smpl}}$ & $\left\langle\left\langle N_{\mathrm{a}}^{\mathrm{T}}(L)\right\rangle\right\rangle_{\mathrm{smpl}}$ \\
\hline 1 & $(0.238085 \pm 0.000067)$ & $\times 10^{2}$ & 13957843.0 & 557850.5 \\
\hline 2 & $(0.569663 \pm 0.000272)$ & $\times 10^{3}$ & 17375161.1 & 553892.4 \\
\hline 3 & $(0.136574 \pm 0.000096)$ & $\times 10^{5}$ & 20943597.7 & 618879.1 \\
\hline 4 & $(0.32779 \pm 0.00026)$ & $\times 10^{6}$ & 25154518.3 & 699008.8 \\
\hline 5 & $(0.78765 \pm 0.00079)$ & $\times 10^{7}$ & 29199658.1 & 779763.9 \\
\hline 6 & $(0.18980 \pm 0.00025)$ & $\times 10^{9}$ & - & - \\
\hline 7 & $(0.45699 \pm 0.00064)$ & $\times 10^{10}$ & - & - \\
\hline 8 & $(0.11010 \pm 0.00017)$ & $\times 10^{12}$ & - & - \\
\hline 9 & $(0.26527 \pm 0.00049)$ & $\times 10^{13}$ & - & - \\
\hline 10 & $(0.6401 \pm 0.0015)$ & $\times 10^{14}$ & - & - \\
\hline
\end{tabular}

Table V. Results on $\left\langle\left\langle Q_{\mathrm{T}\{\eta\}}(L)\right\rangle\right\rangle_{\mathrm{smpl}}$ for $L=1,2, \cdots, 10$ and $\left\langle\left\langle N_{\mathrm{b}}^{\mathrm{T}}(L)\right\rangle\right\rangle_{\mathrm{smpl}}$ and $\left\langle\left\langle N_{\mathrm{a}}^{\mathrm{T}}(L)\right\rangle\right\rangle_{\mathrm{smpl}}$ for $(L=1,2, \cdots, 5)$ in the $S_{z}=1$ case, obtained for the 36-site triangular lattice from a translational invariant trial state $\left|\psi_{\mathrm{T}}\left(S_{z}=1\right)\right\rangle$. Parameters are $\epsilon=0.02$ and $n_{\mathrm{smpl}}=88$ up to $L=5$, and $\epsilon=0.03$ and $n_{\mathrm{smpl}}=180$ for $L \geq 6$. 


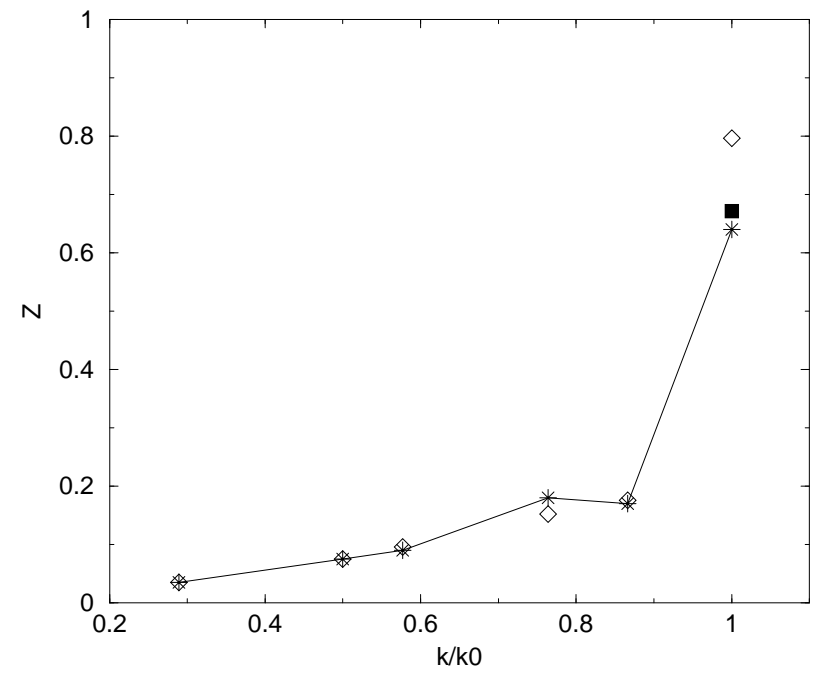

Fig. 2. The static structure factor $Z \equiv 8 F(\mathbf{k}) /\left(N_{\mathrm{s}}+6\right)$ for the 36 -site system versus $k / k_{0}$, where $\pm \mathbf{k}_{0}$ are the two wave vectors of the corners of the crystal Brillouin zone, $k \equiv|\mathbf{k}|$ and $N_{\mathrm{s}}(=36)$ is the number of sites. Asterisks combined by lines to guide eyes are data from ref. 7. Our results obtained by 8 samples of $F_{6}(\mathbf{k})$ with $\epsilon=0.03$ are plotted by open diamonds. A filled square is the value calculated by $F\left(\mathbf{k}_{0}\right)=4.5 \mathcal{M}^{2} / N_{\mathrm{s}}$, where $\mathcal{M}^{2}$ denotes the squared sublattice magnetization. 